\title{
Manifestaciones bucales en pacientes con insuficiencia renal crónica en hemodiálisis
}

\author{
Oral manifestations in patients with chronic renal failure in hemodialysis
}

María Pía Lecca Rojas ${ }^{1, b}$, Jonathan Meza Mauricio ${ }^{1, a}$, Katty Rios Villasis ${ }^{1, c}$

\section{RESUMEN}

Objetivos: Identificar las principales manifestaciones bucales en pacientes con insuficiencia renal crónica en hemodiálisis del Hospital Alberto Sabogal Sologuren (Bellavista, Callao, Perú) durante el periodo de julioagosto 2013. Material y métodos: Se examinaron 119 pacientes de ambos sexos, realizándoseles una anamnesis y luego, con la ayuda de una linterna, espejos bucales y bajalenguas, se les examinó la cavidad bucal de manera minuciosa. Resultados: Los pacientes examinados presentaron: lengua saburral 88,2\%, agrandamiento gingival $63 \%$, sangrado gingival $55,5 \%$, caries dental $85,7 \%$, cálculo dental $80,7 \%$, perdida de inserción dental $72,3 \%$, xerostomía $84 \%$, halitosis $66,4 \%$ y disgeusia $55,5 \%$. Se estudió la relación de las manifestaciones bucales según el tiempo de hemodiálisis, encontrándose que existió una asociación estadísticamente significativa en la mayoría de las lesiones (prueba de Chi Cuadrado; $\mathrm{p}<0,05$ ). Conclusión: Los pacientes con insuficiencia renal crónica en tratamiento de hemodiálisis, presentaron múltiples manifestaciones bucales propias de la enfermedad y también como consecuencia del tratamiento aplicado, donde se ven afectados los tejidos blandos, tejidos duros y glándulas salivales.

PALABRAS CLAVE: Insuficiencia renal crónica, manifestaciones bucales, xerostomía. (DeCS, BIREME)

\section{SUMMARY}

Objective: To identify the main oral manifestations in patients with chronic renal failure on hemodialysis of

\footnotetext{
${ }^{1}$ Facultad de Odontología, Universidad Inca Garcilaso de la Vega. Lima, Perú.

a Bachiller en Estomatología.

${ }^{\text {b }}$ Cirujano Dentista.

c Magíster en Estomatología.
} 
Alberto Sabogal Sologuren Hospital (Bellavista,Callao, Perú) during the period from July to August 2013. Methods: 119 patients of both sexes were examined performing an anamnesis and then with the help of a flashlight, mouth mirrors and tongue depressors, their buccal cavity were thoroughly examined. Results: The patients examined results were: furred tongue $88.2 \%$, gingival enlargement $63 \%$, gingival bleeding $55.5 \%$, dental caries $80.7 \%$, dental calculus $80.7 \%$, dental insertion loss $72.3 \%$, xerostomia $84 \%$, halitosis $66.4 \%$ and dysgeusia $55.5 \%$. The relationship of the oral manifestations by the time of hemodialysis was studied, finding that there was a statistically significant association in most lesions (chi square test, $p<0.05$ ). Conclusion: The patients with chronic renal failure undergoing hemodialysis showed multiple own oral manifestations of the disease and also as a result of the treatment applied, which affected soft tissues, hard tissues and salivary glands.

\section{KEYWORDS: Renal insufficiency chronic, oral manifestations, xerostomia. (MeSH, NLM)}

\section{INTRODUCCION}

La insuficiencia renal crónica (IRC) es una enfermedad de etiología múltiple, donde se produce una disminución lenta, progresiva e irreversible de la función renal, la cual juega un papel importante en nuestro organismo, eliminando sustancias nocivas y productos de desecho (1).

La prevalencia de la IRC se ha incrementado con el tiempo. En la actualidad, se considera una pandemia que afecta aproximadamente al $10 \%$ de la población adulta en diferentes partes del mundo, afectando mayormente a hombres que a mujeres $(2,3)$, debido a que se encuentra asociada a diferentes patologías sistémicas crónicas como la hipertensión arterial, la diabetes mellitus, entre otras (2).

La IRC terminal es la etapa final de la enfermedad que lleva al paciente a la hemodiálisis (4), empeorando su calidad de vida y provocando muchas consecuencias en distintas partes del organismo (5), entre ellas: las manifestaciones bucales (6,7). Éstas, pueden ser propias de la enfermedad o producto de la medicación que recibe el paciente para tratarla, produciendose alteraciones en el sistema estomatognático (6). La mayoría de pacientes con IRC presentan una higiene bucal deficiente, lo cual puede complicar aun más la enfermedad sistémica de base (7).

El $90 \%$ de pacientes con IRC presentan signos y síntomas bucales, que se presentan en los tejidos duros, en los tejidos blandos (8-11) y en las glándulas salivales (12).
En los tejidos blandos se puede encontrar: agrandamiento gingival (12-15), gingivitis $(10,16)$, sangrado gingival $(8,17)$, palidez de la mucosa $(7,9)$, petequias y equimosis $(9)$, lengua saburral $(13,18,19)$, úlceras bucales $(6,9,20)$, candidiasis $(17,21,22)$, queilitis angular $(12,23,24)$. En los tejidos duros se puede observar: placa bacteriana y cálculo dental $(17,25,26)$, caries dental (7), erosión dental $(8,10,11)$, hipoplasia del esmalte $(6,11,27)$, pérdida de inserción y movilidad dental $(10,13)$ y disfunción temporomandibular $(21,28)$. A nivel de glándulas salivales se puede observar: xerostomía $(8,17,18)$, disgeusia $(8,9,12)$ y halitosis $(6,9,17)$.

El objetivo del presente trabajo fue identificar las manifestaciones bucales que presentan los pacientes con IRC en hemodiálisis, a fin de recomendar un mejor cuidado preventivo y así mejorar su calidad de vida.

\section{MATERIAL Y MÉTODOS}

Estudio observacional, prospectivo, transversal y descriptivo. Previamente se solicitó la autorización a la Oficina de Grados y Títulos de la Universidad Inca Garcilaso de la Vega y al Comité de Capacitación, Investigación y Docencia del Hospital Alberto Sabogal Sologuren (Bellavista, Callao). Se utilizó un muestreo por conveniencia. La muestra estuvo conformada por 119 pacientes que acudieron al Centro de Hemodiálisis del Hospital Alberto Sabogal Sologuren, en los meses de julio-agosto 2013. Los criterios de inclusión fueron: pacientes mayores de 18 años de edad con IRC en hemodiálisis, sin retardo 
mental y que colaboren al examen clínico. Se les brindo la información necesaria y voluntariamente dieron su aprobación a través del Consentimiento Informado.

Los pacientes que decidieron formar parte del estudio fueron evaluados en el momento en que se les realizaba la hemodiálisis. Primero, se realizó la anamnesis y luego, con la ayuda de una linterna, espejos bucales y bajalenguas, se les examinó la cavidad bucal de manera minuciosa. Se tomaron fotografías y se realizó el registro correspondiente en la ficha de recolección de datos, de todas las manifestaciones bucales que presentó cada paciente.

Para el análisis estadístico de los resultados se utilizó el programa SPSS en su versión de acceso para Windows. Se fijó un grado de significancia de 0,05 que corresponde a un intervalo de confianza del $95 \%$. Los datos fueron analizados mediante estadística analítica y descriptiva.

\section{RESULTADOS}

De los 119 pacientes evaluados, que entre julio y agosto del 2013 acudieron al Centro de Hemodiálisis del Hospital Alberto Sabogal Sologuren, el 58\% $(n=69)$ perteneció al sexo masculino y el $42 \%(n=50)$ al sexo femenino, teniendo un promedio de edad de 53,13 $\pm 17,00$ años; la edad mínima fue 20 y la máxima de 89 años.

La prevalencia de manifestaciones bucales en pacientes con IRC en hemodiálisis, fue la siguiente: agrandamiento gingival $63 \% \quad(\mathrm{n}=75)$, sangrado gingival 55,5\% ( $\mathrm{n}=66)$, gingivitis 49,6\% $(\mathrm{n}=59)$, palidez de la mucosa $52,1 \%(n=62)$, petequias $23,5 \%$ $(\mathrm{n}=28)$, equimosis $13,4 \%(\mathrm{n}=16)$, lengua saburral $88,2 \% \quad(n=105)$, úlceras bucales $32,8 \% \quad(n=39)$, ardor en la boca $52,9 \%(\mathrm{n}=63)$, queilitis angular $55,5 \% \quad(n=66)$, candidiasis $19,3 \% \quad(n=23)$, caries dental $85,7 \%(n=102)$, erosión dental 58\% $(n=69)$, hipoplasia del esmalte $12,6 \%(n=15)$, pérdida de inserción dental 72,3\% ( $\mathrm{n}=86)$, cálculo dental $80,7 \%$ $(\mathrm{n}=96)$, movilidad dental 58\% $(\mathrm{n}=69)$, disfunción temporomandibular 56,3\% ( $\mathrm{n}=67)$, xerostomía $84,0 \%$ $(\mathrm{n}=100)$, disgeusia $55,5 \%(\mathrm{n}=66)$ y halitosis $66,4 \%$ $(n=79)$ (Tabla 1).
Respecto a la prevalencia de manifestaciones bucales en los tejidos blandos en pacientes con IRC, según el tiempo de hemodiálisis, se observó que existió asociación estadísticamente significativa entre agrandamiento gingival, sangrado gingival, gingivitis, petequias, equimosis, úlceras bucales, ardor en la boca, queilitis angular y tiempo de hemodiálisis (prueba de Chi Cuadrado; $\mathrm{p}<0,05$ ). No se encontró asociación estadísticamente significativa entre palidez de la mucosa, lengua saburral, candidiasis, y tiempo de hemodiálisis (prueba de Chi Cuadrado; $\mathrm{p}>0,05$ ) (Tabla 2).

Al evaluar la prevalencia de manifestaciones bucales en los tejidos duros en pacientes con IRC, según el tiempo de hemodiálisis, se observó asociación estadísticamente significativa entre caries dental, erosión dental, hipoplasia del esmalte, pérdida de inserción dental, cálculo dental, movilidad dental, disfunción temporomandibular y tiempo de hemodiálisis (prueba de Chi Cuadrado; $\mathrm{p}<0,05$ ) (Tabla 3).

Tabla 1. Prevalencia de manifestaciones bucales en pacientes con IRC, en hemodiálisis.

\begin{tabular}{lc}
\hline \multicolumn{1}{c}{ Manifestaciones Bucales } & n (\%) \\
\hline Agrandamiento gingival & $75(63,0)$ \\
Sangrado gingival & $66(55,5)$ \\
Gingivitis & $59(49,6)$ \\
Palidez de la mucosa & $62(52,1)$ \\
Petequias & $28(23,5)$ \\
Equimosis & $16(13,4)$ \\
Lengua saburral & $105(88,2)$ \\
Úlceras bucales & $39(32,8)$ \\
Queilitis angular & $66(55,5)$ \\
Candidiasis & $23(19,3)$ \\
Caries dental & $102(85,7)$ \\
Erosión dental & $69(58,0)$ \\
Hipoplasia del esmalte & $15(12,6)$ \\
Pérdida de inserción dental & $86(72,3)$ \\
Cálculo dental & $96(80,7)$ \\
Movilidad dental & $69(58,0)$ \\
Xerostomía & $100(84,0)$ \\
Disgeusia & $66(55,5)$ \\
Halitosis & $79(66,4)$ \\
\hline
\end{tabular}


Respecto a la prevalencia de manifestaciones bucales en las glándulas salivales en pacientes con IRC, según el tiempo de hemodiálisis, se observó asociación estadísticamente significativa entre xerostomía, halitosis y disgeusia con el tiempo de hemodiálisis (prueba de Chi Cuadrado; $\mathrm{p}<0,05$ ) (Tabla 4).

\section{DISCUSIÓN}

De acuerdo a los resultados obtenidos se demostró que los pacientes con IRC en hemodiálisis, presentaron alguna manifestación bucal debido a su estado sistémico o como consecuencia del tratamiento.
En los tejidos blandos se observaron diversas manifestaciones bucales, entre ellas la de mayor prevalencia fue la lengua saburral $(88,2 \%)$ que se da probablemente como consecuencia de la restricción de líquidos que tienen los pacientes; pero también juega un papel importante la higiene bucal. El agrandamiento gingival se presentó en el $63 \%$ de los pacientes, probablemente como consecuencia del tratamiento farmacológico. Al respecto, Belazelkovska y col. (12) encontraron lengua saburral en el $100 \%$ de sus pacientes y con relación al agrandamiento gingival, ninguno de sus pacientes presentó. Mientras que Kaushik y col. (17) observaron

Tabla 2. Prevalencia de manifestaciones bucales en tejidos blandos en pacientes con IRC,

\begin{tabular}{lccccc}
\multicolumn{5}{c}{ por meses y según tiempo de hemodiálisis. } \\
\hline \multicolumn{1}{c}{ Tejidos Blandos } & $\mathbf{1 - 2 4}$ & $\mathbf{2 5 - 4 8}$ & $\mathbf{4 9 - 9 6}$ & $\mathbf{9 7 - 1 6 8}$ & \\
\hline Agrandamiento gingival & $\mathbf{n}(\mathbf{\%})$ & $\mathbf{n}(\mathbf{\%})$ & $\mathbf{n}(\mathbf{\%})$ & $\mathbf{n}(\mathbf{\%})$ & $\boldsymbol{P}$ \\
Sangrado gingival & $11(9,2)$ & $25(21,0)$ & $22(18,5)$ & $17(14,3)$ & 0,000 \\
Gingivitis & $8(6,7)$ & $23(19,3)$ & $19(16,0)$ & $16(13,4)$ & 0,029 \\
Palidez de la mucosa & $13(10,9)$ & $16(13,4)$ & $19(16,0)$ & $11(9,2)$ & 0,029 \\
Petequias & $20(16,8)$ & $18(15,1)$ & $14(6,7)$ & $10(8,4)$ & 0,989 \\
Equimosis & $1(0,8)$ & $5(4,2)$ & $13(7,6)$ & $9(7,6)$ & 0,000 \\
Lengua saburral & $3(2,5)$ & $2(1,7)$ & $6(5,0)$ & $5(4,2)$ & 0,081 \\
Úlceras bucales & $32(26,9)$ & $29(24,4)$ & $27(22,7)$ & $17(14,3)$ & 0,155 \\
Queilitis angular & $3(2,5)$ & $9(7,6)$ & $12(10,1)$ & $15(12,6)$ & 0,000 \\
Candidiasis & $9(7,6)$ & $20(16,8)$ & $20(16,8)$ & $17(14,3)$ & 0,000 \\
\hline
\end{tabular}

Tabla 3. Prevalencia de manifestaciones bucales en tejidos duros en pacientes con IRC, por meses y según tiempo de hemodiálisis.

\begin{tabular}{lccccc}
\hline & $\mathbf{1 - 2 4}$ & $\mathbf{2 5 - 4 8}$ & $\mathbf{4 9 - 9 6}$ & $\mathbf{9 7 - 1 6 8}$ & \\
Tejidos duros & $\mathbf{n ~ ( \% )}$ & $\mathbf{n}(\mathbf{\%})$ & $\mathbf{n}(\mathbf{\%})$ & $\mathbf{n}(\mathbf{\%})$ & $\boldsymbol{P}$ \\
\hline Caries dental & $26(21,8)$ & $31(26,1)$ & $26(21,8)$ & $19(16,2)$ & 0,001 \\
Erosión dental & $8(6,7)$ & $26(21,8)$ & $18(15,1)$ & $17(14,3)$ & 0,000 \\
Hipoplasia del esmalte & $0(0,0)$ & $3(2,5)$ & $6(5,0)$ & $6(5,0)$ & 0,003 \\
Pérdida de inserción dental & $18(15,1)$ & $29(24,4)$ & $22(18,5)$ & $17(14,3)$ & 0,000 \\
Cálculo dental & $21(17,6)$ & $30(25,2)$ & $26(21,8)$ & $19(16,0)$ & 0,000 \\
Movilidad dental & $14(11,8)$ & $20(16,8)$ & $20(16,8)$ & $15(12,6)$ & 0,004 \\
\hline
\end{tabular}


Tabla 4. Prevalencia de manifestaciones bucales en glándulas salivales en pacientes con IRC, por meses y según tiempo de hemodiálisis.

\begin{tabular}{lccccc}
\hline \multicolumn{1}{c}{ Glándulas salivales } & $\mathbf{1 - 2 4}$ & $\mathbf{2 5 - 4 8}$ & $\mathbf{4 9 - 9 6}$ & $\mathbf{9 7 - 1 6 8}$ & \\
\hline Xerostomía & $\mathbf{n ~ ( \% )}$ & $\mathbf{n ~ ( \% )}$ & $\mathbf{n ~ ( \% )}$ & $\mathbf{n ~ ( \% )}$ & $\boldsymbol{P}$ \\
Disgeusia & $24(20,2)$ & $31(26,1)$ & $25(21,0)$ & $20(16,8)$ & 0,000 \\
Halitosis & $11(9,2)$ & $22(18,5)$ & $18(15,1)$ & $15(12,6)$ & 0,000 \\
\hline
\end{tabular}

que el $10 \%$ de su población presentó agrandamiento gingival y el $23 \%$ lengua saburral.

Respecto a queilitis angular, los resultados encontrados en el presente estudio 55,5\%, coincidieron con los reportados por Belazelkovska y col. (12) $63,33 \%$, posiblemente relacionado al elevado porcentaje de xerostomía que presentaron los pacientes en ambos estudios.

La prevalencia de sangrado gingival encontrada en el presente estudio 55,5\%, fue mayor que lo reportado por Kaushik y col. (17) $24 \%$, Cedeño y col. (8) $15,8 \%$ y Rebolledo y col. (7) 10,7\%. Algunos factores pueden haber intervenido en la diferencia de los resultados, tales como: el estado sistémico de los pacientes, la cantidad y frecuencia de dosis de anticoagulantes administradas, los cuidados bucales y el momento en que se examinó a los pacientes. En el presente estudio fue durante el tratamiento de hemodiálisis, en el caso de los otros estudios, no se reporta el momento de la evaluación clínica bucal.

La palidez de la mucosa que presentaron los pacientes con IRC en hemodiálisis, se debe probablemente al sangrado debido a la anemia que presenta la mayoría de ellos. En los pacientes evaluados en el presente estudio se encontró que el $52,1 \%$ presentó esta manifestación, porcentaje mayor que el encontrado por Rebolledo y col. (7) 39,25\%, pero menor que lo observado por Cedeño y col. (8) $75,6 \%$, Patil y col. (9) $87 \%$ y Belazelkovska y col. (12) $83,33 \%$.

La gingivitis en estos pacientes, va a depender del estado sistémico en que se encuentren y de la higiene bucal que presenten. La prevalencia de esta manifestación bucal en el presente estudio fue de
49,6\%, coincidiendo con lo observado por Díaz y col. (6) $50 \%$. Mientras que Muñoz y col. (13) encontraron una prevalencia mayor, $78 \%$.

La candidiasis bucal se produce debido al estado de inmunosupresión en que se encuentra el paciente dializado, si a esto se le agrega la xerostomía que presentan y su condición de adulto mayor (en algunos casos), los predispone a desarrollar esta manifestación bucal. En el presente estudio se encontró que el $19,3 \%$ de los pacientes evaluados presentaba esta manifestación bucal, porcentaje más elevado de lo observado por Belazelkovska y col. (12) $10 \%$ y Muñoz y col. (13) 6,54\%.

En los tejidos duros, la manifestación de mayor prevalencia fue la caries dental $85,7 \%$, coincidiendo con Rebolledo y col. (7) 83,9\% y Muñoz y col. (13) 64\%. Por otro lado Díaz y col. (6) presentaron un porcentaje más elevado $93 \%$. Esto puede ser debido a que dicho estudio fue realizado en pacientes niños, donde la mayoría presentaba hipoplasia del esmalte y por ende mayor riesgo a desarrollar lesiones de caries dental. La segunda manifestación que obtuvo un resultado elevado en el presente estudio, fue el cálculo dental $80,7 \%$, lo cual coincide con el estudio de Souza y col. (25) $86,7 \%$ y el de Rebolledo y col. (7) $61,6 \%$.

Los pacientes con IRC se encuentran predispuestos a formar cálculo dental, debido al aumento de los niveles de urea en saliva, fósforo y por las grandes cantidades de carbonato de calcio que algunos ingieren como parte de su tratamiento.

La pérdida de inserción y movilidad dental se dan por alteraciones que se producen en el metabolismo óseo y mineral, al verse afectado el balance calcio- 
fosforo y la mineralización. En el presente estudio se observó una prevalencia de pérdida de inserción dental de $72,3 \%$ y movilidad dental de $58 \%$, resultados mayores que los encontrados por Rebolledo y col. (7) $12,5 \%$ y $28,5 \%$ respectivamente.

La erosión dental se relaciona, muchas veces, con las regurgitaciones que presentan los pacientes debido a los medicamentos, dosis elevada de urea que presentan o como consecuencia del tratamiento de hemodiálisis, los resultados que se obtuvieron en el presente estudio (58\%) no coinciden con los de Cedeño y col. (8) 10,9\%, cuyos pacientes presentaron un porcentaje mucho más bajo.

La hipoplasia del esmalte es una alteración que se produce en el desarrollo dentinario como un defecto en la producción de la matriz del esmalte. En el presente estudio se observó una prevalencia de $12,6 \%$, igual a lo observado por Patil y col. (9) $12 \%$, pero diferente a lo observado por Díaz y col. (6), quienes evaluaron sólo niños, obteniendo en esta población infantil una prevalencia de $77 \%$.

Respecto a las lesiones de glándulas salivales, tuvo mayor prevalencia la xerostomía (84\%), seguida de la halitosis $(66,4 \%)$ y disgeusia $(55,5 \%)$. Estas manifestaciones se producen debido a que los pacientes con insuficiencia renal crónica en hemodiálisis tienen restricción de la ingesta de líquidos; y si a eso se suman los daños que pueden tener en las glándulas salivales, debido a los altos niveles de urea que presentan cuando no están controlados, se encuentran aun más predispuestos a desarrollar dichas manifestaciones. La prevalencia de xerostomía presentada coincide con el estudio de Patil y col. (9) 91\% y con el de Díaz y col. (6) 93\%. Por otro lado, Belazelkovska y col. (12) $(66,66 \%)$ y Cedeño y col. (8) $(53,6 \%)$ reportaron una prevalencia más baja.

La prevalencia de halitosis fue de $66,4 \%$ al igual que lo observado por Souza y col. (25) (60,7\%) y Belazelkovska y col. (12) $(56,66 \%)$, mientras que los estudios de Kaushik y col. (17) y Patil y col. (9) presentaron prevalencias menores, $43 \%$ y $34 \%$ respectivamente.

Con relación a la disgeusia se encontró una prevalencia de $55,5 \%$ coincidiendo con lo encontrado por Cedeño y col. (8) $51,2 \%$ y Patil y col. (9) $42 \%$; mientras que Kaushik y col. (17) presentaron prevalencias más bajas $37 \%$, coincidiendo con Belazelkovska y col. (12) 33,33\%.

En los estudios de Cedeño y col. (8), Belazelkovska y col. (12), Kaushik y col. (17), Souza y col. (25) no se evidencia asociación directa de las manifestaciones bucales con el tiempo de hemodiálisis, por lo cual se decidió observar si a mayor tiempo de hemodiálisis los pacientes presentaban mayor cantidad de manifestaciones bucales. Los pacientes evaluados cursaban desde 1 a 168 meses realizándose hemodiálisis, periodo que fue dividido en intervalos de: 1-24, 25-48, 49-96 y 97-168 meses. Se pudo observar que hubo una asociación estadísticamente significativa entre las manifestaciones bucales y el tiempo de hemodiálisis (Chi Cuadrado; $\mathrm{p}<0,05$ ). Es decir, pacientes que llevaban más tiempo en tratamiento de hemodiálisis, estaban más predispuestos a desarrollar diversas manifestaciones bucales, a diferencia de los que recién comenzaban su tratamiento.

Este estudio es importante porque permite conocer las alteraciones bucales que presentan los pacientes con IRC en hemodiálisis. Este conocimiento permitirá orientar a los odontólogos, mejorando las condiciones bucales de éstos pacientes, y por ende brindarles una mejor calidad de vida.

\section{CONCLUSIONES}

La mayoría de los pacientes evaluados con IRC y que se encontraron en hemodiálisis, presentaron múltiples manifestaciones bucales. Si a esto le agregamos que tienen un tiempo prolongado en hemodiálisis, se vuelven mucho más predispuestos a desarrollar algún tipo de manifestación bucal, lo cual puede ser propio de la enfermedad o del tratamiento que reciben.

Las manifestaciones bucales en pacientes con IRC en hemodiálisis, con mayor prevalencia en tejidos blandos fueron: lengua saburral, agrandamiento gingival, sangrado gingival, queilitis angular, palidez de la mucosa; en los tejidos duros: caries dental, 
cálculo dental, pérdida de inserción dental, movilidad dental; y en las glándulas salivales: xerostomía, halitosis y disgeusia.

\section{Correspondencia:}

\section{María Pía Lecca Rojas}

Cacique Umachiri №170 - San Miguel. Lima, Perú. Correo electrónico: pia_341@hotmail.com

\section{REFERENCIAS BIBLIOGRÁFICAS}

1. Soriano S. Definición y clasificación de los estadios de la enfermedad renal crónica. Prevalencia: claves para el diagnóstico precoz. Factores de riesgo de la enfermedad renal crónica. Nefrología. 2004; 24(S6): 27-34.

2. Ávila-Saldivar M. Enfermedad renal crónica: prevención y detección temprana en el primer nivel de atención. Med Int Mex. 2013; 29(2):148-153.

3. Coresh J, Selvin E, Stevens L, Manzi J, Kusek JW, Eggers P. et al. Prevalence of chronic kidney disease in the United States. JAMA. 2007; 298(17):2038-47.

4. Flores J, Alvo M, Borja H, Morales J, Vega J, Zúñiga C. et al. Enfermedad renal crónica: clasificación, identificación, manejo y complicaciones. Rev Med Chile. 2009; 137(1):137-77.5.

5. Murphree D, Thelen S. Chronic kidney disease in primary care. J Am Board Fam Med. 2010; 23(4):542-550.

6. Díaz A, Flores F, Hernández J, Pérez C, Jiménez C. Alteraciones bucodentales en niños con insuficiencia renal crónica y trasplante renales. Acta Odontol Venez. 2010; 48(2):1-11.

7. Rebolledo C, Carmona L, Carbonelle M. Salud oral en pacientes con insuficiencia renal crónica hemodializados después de la aplicación de un protocolo estomatológico. Av Odontoestomatol. 2012; 28(2):77-87.

8. Cedeño J, Rivas N, Tuliano R. Manifestaciones bucales en pacientes con enfermedad renal crónica terminal bajo tratamiento de hemodiálisis y su manejo en cirugía bucal. VITAE. 2011; 46(1):1-7.

9. Patil S, Khaandelwal S, Doni B, Rahuman F, Kaswan $\mathrm{S}$. Oral manifestations in chronic renal failure patients attending two Hospital in North Karnataka, Indian. Oral Health Dent Manag. 2012; 11(3):100-6.

10. Jover A, Bagán J, Jiménez Y, Poveda R. Dental management in renal failure: patients on dialysis. Med Oral Patol Oral Cir Bucal. 2008; 13(7):E419-26.

11. Haider S, Tanwir F, Monin I. Oral aspects of chronic renal failure. Pak Oral Dental J. 2013; 33(1): 87-90.

12. Belazelkovska A, Popovska M, Spasovski G, et al. Oral clinical findings in patients with chronic renal failure. Balk J Stom. 2013; 17(1):37-43.

13. Muñoz E, Restrepo C, Chacón J. Caracterización en salud oral y hábitos de higiene oral en pacientes con enfermedad renal crónica. Act Med Col. 2011; 36(4):173-80.

14. Díaz A, Arevalo L, Fonseca M. Agrandamiento gingival inducido por nifedipina: reporte de un caso. Duazari. 2009; 6(1):51-5.

15. Alberto G, Zayas R, Fragoso R, Cuairan V, Hernández A. Manejo estomatológico en pacientes con insuficiencia renal crónica: presentación de caso. Rev Odont Mex. 2009; 13(3):171-6.

16. Marakoglu I, Gursoy U, Demier S, Sezer H. Periodontal status of chorinic renal failure patients receiving hemodialysis. Yonsei Med J. 2003; 44(4):648-52.

17. Kaushik A, Reddy S, Umesh L, Devi B, Santana N, Rakesh N. Oral and salivary changes among renal patients undergoing hemodialysis: a cross-sectional study. Indian J Nephorol. 2013; 23(2):125-9.

18. Espitia S, Castillo L, Carbonell B, Mórelo A, Pacheco M. Asociación entre la disminución de los niveles de flujo salival y xerostomía en pacientes con insuficiencia renal crónica hemodializados de la unidad renal nefrología LTDA, de la ciudad de Santa Marta. Duazary. 2007; 4(2):100-11.

19. De la Rosa E, Mondragón A, Aranda S, Bustamante M. Oral mucosa symptoms, signs and lesions, in end stage renal diasease and non-end stage renal disease diabetic patients. Med Oral Patol Oral Cir Bucal. 2006; 11(1):E467-73.

20. Sudarshan R, Annigeri R, Mamatha G, Vijayabala S. Uremic stomatitis. Contemp Clin Dent. 2012; 3(1):113-5.

21. Lovera K, Delgado E, Bereni L, Gay C. El paciente con insuficiencia renal en la práctica odontológica. RCOE. 2000; 5(5):521-31.

22. Aguirre J. Candidiasis orales. Rev Iberoam Micol. 2002; 19(1):17-21.

23. Sapp P, Eversole L, Wysocki G. Enfermedades hematológicas. En: Sapp P, Eversole L, Wysocki G. (Editores). Patología oral y maxilofacial contemporánea. 2 ed. Madrid: Harcourt Brace; 1998. 
24. García E, Blanco A, Rodríguez L, Reyes D, Sotres J. Queilitis. Revisión bibliográfica. Rev Cubana Estomatol. 2004; 41(2):1-10.

25. Souza C, Braosila A, Luczyszyn S, et al. Oral health Brazilian patients with chronic renal disease. Rev Med Chile. 2008; 136(6):741-6.

26. Díaz A, Fonseca M, Parra C. Calculo dental una revisión de literatura y presentación de una condición inusual. Acta Odontol Venez. 2011; 49(3):1-11.

27 Acosta M. Hipoplasia dental asociada a enfermedad renal. Revista Latinoamericana de Ortodoncia y
Odontopediatría. 2008; 15(3):97-107.

28. Peñon P, Grau I, Sarracent H. Caracterización clínica del síndrome de disfunción temporomandibular en el Hospital Universitario Miguel Enríquez. Rev Cubana de Estomatología. 2011; 48(4):371-81.

Recibido: 18/01/2014 Aceptado: 15/04/2014 